\title{
Avaliação da eficácia de vacina autóctone de Streptococcus agalactiae inativado aplicada por banho de imersão em tilápia do Nilo (Oreochromis niloticus)
}

\section{Effectiveness of homologous inactivated Streptococcus agalactiae vaccine by immersion bath in Nile tilapia (Oreochromis niloticus)}

\author{
Elaine Longhi ${ }^{1}$; Lucienne Garcia Pretto-Giordano ${ }^{2 *}$; Ernst Eckehardt Müller ${ }^{3}$
}

\section{Resumo}

O grupo das tilápias é um dos mais importantes entre os peixes cultivados. O sistema de cultivo mais utilizado é o intensivo, caracterizado por alta taxa de estocagem de peixes tornando-os susceptíveis às doenças infecciosas. A estreptococose é uma das enfermidades que causam grandes prejuízos econômicos. O objetivo deste trabalho foi testar uma vacina de Streptococcus agalactiae inativado aplicada por banho de imersão (b.i) e desafio com cepa homóloga. Foram utilizados 421 tilapia do Nilo (Oreochromis niloticus) com peso médio de 38,38 gramas, distribuídas em dois tratamentos (T1 e T2) e um grupo controle. No T1, os peixes foram vacinados com uma dose da vacina por b.i., com concentração de $5,4 \times 10^{8} \mathrm{UFC} \mathrm{mL}^{-1}$. O T2 recebeu duas doses da mesma vacina por b.i., com intervalo de 25 dias. $\mathrm{O}$ grupo controle recebeu água ultrapura por b.i. Os peixes foram desafiados por

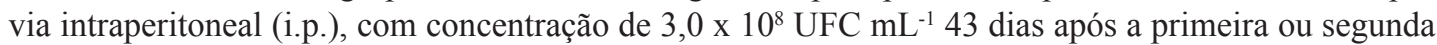
dose da vacina, de acordo com o tratamento. Os peixes dos tratamentos e controle foram monitorados durante 16 dias após o desafio. Comparativamente ao grupo controle os resultados obtidos para o T1 foram: $\mathrm{p}=0,0805, \mathrm{RR}=0,79$ (IC 95\%: 0,61-1,01) e RPS $=21 \%$. Para o T2 foram: $\mathrm{p}=0,0296, \mathrm{RR}=$ 0,74 (IC 95\%: 0,56-0,96) e RPS $=26 \%$. A mortalidade após o desafio foi de 57 peixes $(40,71 \%)$ no T1, $51(38,06 \%)$ no T2, e $76(51,7 \%)$ no grupo controle. Não houve diferença significativa entre T1 e T2 e p=0,7445. Este resultado permite concluir que a vacina testada por b.i. com uma e duas doses apresentou baixa eficácia na imunização das tilápias.

Palavras-chave: Estreptococose, imunização, peixes

\begin{abstract}
Tilapia is the most important group between cultivated fish. Intensive handling systems are characterized by high rate of stocking of fish making them susceptible to infectious diseases. The streptococcosis is one of the diseases that cause great economic losses. The objective of the study was to test a vaccine administered by immersion bath (b.i.) against Streptococcus agalactiae and challenge with homologous strain. For this study, 421 Nile tilapia (Oreochromis niloticus) with an average weight of 38.38 grams were used. They were divided into two treatments (T1 and T2) and a control group. At T1, the fish were vaccinated with one dose of vaccine by b.i., with a concentration of $5.4 \times 10^{8} \mathrm{CFU} \mathrm{mL}^{-1}$. The $\mathrm{T} 2$ received two doses of vaccine by b.i., with an interval of 25 days. The control group received a bath of ultrapure water. The fish were challenged by intraperitoneal (i.p.) with a concentration of $3.0 \times 10^{8} \mathrm{CFU} \mathrm{mL}^{-1} 43$

1 Discente do curso de Pós-graduação em Ciência Animal, Universidade Estadual de Londrina, UEL, Londrina, PR. E-mail: elainelonvet@hotmail.com

2 Médica Veterinária, Laboratório de Microbiologia Veterinária e Doenças Infecciosas, UEL, Londrina, PR. E-mail: lgiordano@

3 Prof. do Laboratório de Microbiologia Veterinária e Doenças Infecciosas, UEL, Londrina, PR. E-mail:muller@uel.br Autor para correspondência
\end{abstract} uel.br 
days after the first or second dose of vaccine, according to the treatment. The fish were monitored for 16 days after challenge. Compared with the control group results were obtained for T1: $\mathrm{p}=0.0805, \mathrm{RR}=$ 0.79 (95\% CI: $0.61-1.01)$ and RPS $=21 \%$. For T2 were: $\mathrm{p}=0.0296, \mathrm{RR}=0.74(95 \% \mathrm{CI} 0.56$ to 0.96$)$ and RPS $=26 \%$. Mortality after challenge was 57 fish (40.71\%) in T1, $51(38.06 \%)$ at T2, and $76(51.7 \%)$ in the control group. There was no significant difference between $\mathrm{T} 1$ and $\mathrm{T} 2, \mathrm{p}=0.7445$. This result shows that the vaccine tested by b.i. with one and two doses had low efficacy in vaccination of tilapia.

Key words: Streptococcosis, immunization, fish

\section{Introdução}

O grupo das tilápias é um dos mais importantes entre os peixes cultivados. O sistema de cultivo normalmente utilizado é o intensivo, caracterizado por alta taxa de estocagem de peixes e, consequentemente, arraçoamento intensivo, podendo ocorrer diminuição da qualidade da água e estresse dos animais, tornando-os susceptíveis às enfermidades infecciosas (PLUMB, 1997; SHOEMAKER; EVANS; KLESIUS, 2000). As principias espécies de bactérias patogênicas para peixes são Aeromonas hydrophila, Flavobacterium columnare, Edwardisiella tarda, Vibrio vulnificus, Streptococcus iniae e Streptococcus agalactiae (ROBERTS; SOMMERVILLE, 1982; FIGUEIREDO; LEAL, 2008).

Entre as doenças de origem bacteriana que causam maiores problemas, destaca-se a estreptococose por provocar elevados prejuízos para a produção de peixes de água doce e salgada. A doença tem sido relatada como uma das principais em diversos países de destaque na aquicultura, inclusive no Brasil (SALVADOR et al., 2005; PARK et al., 2009).

A estreptococose deve ser considerada como um complexo de doenças similares causadas por diferentes gêneros e espécies de bactérias (Lactococcus garvieae, Lactococccus piscium, S. iniae, S. agalactiae, Streptococcus parauberis e Vagococcus salmoninarum) capazes de lesar o sistema nervoso central (TORANZO et al., 2005). No Brasil, S. agalactiae é a espécie mais isolada em tilápias e os principais sinais clínicos observados são opacidade de córnea, exoftalmia uni ou bilateral, natação errática, letargia, ascite e escurecimento da pele (SALVADOR et al., 2003, 2005; FIGUEIREDO et al., 2006).
As principais medidas utilizadas no controle da estreptococose está relacionada com manejo, alimentação, utilização de antibióticos e produtos químicos. Porém o uso indiscriminado de antibióticos e produtos químicos pode causar poluição e degradação do meio ambiente, além de seleção de cepas resistentes e risco à saúde do consumidor (GRISEZ; OLLEVIER, 1995; SWAIN et al., 2002; KLESIUS; EVANS; SHOEMAKER, 2004; SERRANO, 2005).

O desenvolvimento de novas vacinas e a melhoria nas técnicas de vacinação tem auxiliado o controle de algumas doenças infecciosas na aquicultura (HASTEIN; GUDDING; EVENSEN, 2005). Devido aos benefícios econômicos, as vacinas produzidas a partir de células inativadas são amplamente usadas para a proteção dos peixes frente a diferentes doenças bacterianas (DUMRONGPHOL et al., 2009). Os peixes podem ser imunizados pelas vias intraperitoneal (i.p.), oral (v.o.) e por banho de imersão (b.i.). Esses métodos apresentam vantagens e desvantagens relacionadas ao nível de proteção, efeitos colaterais, praticidade e custo-benefício (GOMES; AFONSO; GARTNER, 2006).

As vacinas estreptocócicas aplicadas por via i.p. apresentam melhores resultados na proteção de tilápias, mas não é de fácil aplicação, é um fator de estresse para os peixes e apresenta maior custo com a mão de obra qualificada. . A aplicação da vacina por b.i. é menos eficaz, mas tem como vantagem o baixo custo e a praticidade, podendo ser utilizado em peixes de menor porte e em grande quantidade (NAKANISHI; KIRYU; OTOTAKE, 2002; EVANS et al., 2004b; PRETTO-GIORDANO et al., 2010b). 
Em 2011, foi aprovada no Brasil a primeira vacina de $S$. agalactiae inativado para tilápias, Aquavac $^{\circledR}$ STREP Sa, de aplicação i.p. que em condições experimentais, apresenta RPS de $84 \%$ nos peixes vacinados e desafiados com $S$. agalactiae por via i.p. (MSD SAÚDE ANIMAL, 2012). O objetivo deste trabalho foi avaliar a eficácia de uma vacina autóctone de $S$. agalactiae inativado aplicada por b.i. em tilápia do Nilo (Oreochromis niloticus) em condições experimentais.

\section{Material e Métodos}

\section{Peixes}

Foram utilizadas 421 tilápia do Nilo (Oreochromis niloticus), com peso médio de 38,38g. O experimento foi conduzido na Estação de
Piscicultura do Departamento de Biologia Animal e Vegetal da Universidade Estadual de Londrina, em Londrina, Paraná.

As tilápias foram distribuídas em caixas de fibra de vidro com capacidade de 500L (Tabela 1), 10 dias antes do início do experimento, para aclimatação. O arraçoamento foi realizado duas vezes ao dia na proporção de $1,5 \%$ do peso vivo, com ração extrusada, com pelets de $2 \mathrm{~mm}$, e $35 \%$ de proteína bruta (FISH 35\%, Extrusada, Cooperativa Integrada $\left.{ }^{\circledR}\right)$. Antes do início do experimento foram necropsiados aleatoriamente nove peixes, colhido material de rim cranial, encéfalo e fígado para exame bacteriológico. As amostras colhidas foram negativas para $S$. agalactiae e/ou outros agentes bacterianos.

Tabela 1. Delineamento experimental da avaliação da eficácia de uma vacina de Streptococcus agalactiae inativado aplicada por banho de imersão (b.i) em tilápia do Nilo (Oreochromis niloticus) e desafiadas por via intraperitoneal (i.p.), em Londrina-PR, 2009.

\begin{tabular}{|c|c|c|c|c|c|c|}
\hline \multirow[t]{2}{*}{ Tratamentos } & \multirow[t]{2}{*}{ Repetições } & \multirow{2}{*}{$\begin{array}{l}\mathrm{N}^{\circ} \text { de } \\
\text { tilápias }\end{array}$} & \multicolumn{2}{|c|}{$\begin{array}{l}\text { Esquema de Vacinação } \\
5,4 \times 10^{8}{\text { UFC } \mathrm{mL}^{-1}}^{-1}\end{array}$} & \multirow{2}{*}{$\begin{array}{c}\text { Desafio } 3,0 \times 10^{8} \\
\text { UFC mL }{ }^{-1}\end{array}$} & \multirow[t]{2}{*}{$\begin{array}{l}\text { Período de } \\
\text { observação }\end{array}$} \\
\hline & & & Dia 0 & Dia 25 & & \\
\hline \multirow{3}{*}{$\mathrm{T} 1$} & 1 & 47 & & \multirow{4}{*}{$1^{\mathrm{a}}$ dose b.i. } & \multirow{4}{*}{$\begin{array}{l}\text { Desafio } \\
0,1 \mathrm{~mL} \text { i.p }\end{array}$} & \multirow{4}{*}{16 dias } \\
\hline & 2 & 47 & & & & \\
\hline & 3 & 46 & & & & \\
\hline Total & 3 & 140 & & & & \\
\hline \multirow{3}{*}{$\mathrm{T} 2$} & 1 & 45 & \multirow{4}{*}{$1^{\mathrm{a}}$ dose b.i. } & \multirow{4}{*}{$2^{\mathrm{a}}$ dose b.i. } & \multirow{4}{*}{$\begin{array}{l}\text { Desafio } \\
0,1 \mathrm{~mL} \text { i.p }\end{array}$} & \multirow{4}{*}{16 dias } \\
\hline & 2 & 45 & & & & \\
\hline & 3 & 44 & & & & \\
\hline Total & 3 & 134 & & & & \\
\hline \multirow{3}{*}{ Controle } & 1 & 49 & & \multirow{4}{*}{$\begin{array}{c}\text { Água } \\
\text { ultrapura } \\
\text { b.i. }\end{array}$} & \multirow{4}{*}{$\begin{array}{l}\text { Desafio } \\
0,1 \mathrm{~mL} \text { i.p }\end{array}$} & \multirow{4}{*}{16 dias } \\
\hline & 2 & 49 & & & & \\
\hline & 3 & 49 & & & & \\
\hline Total & 3 & 147 & & & & \\
\hline
\end{tabular}

Fonte: Elaboração dos autores.

\section{Controle da qualidade da água}

As caixas continham 400L de água de poço semi-artesiano, com fluxo de $3 \mathrm{~L}$ de água/minuto, aeração contínua e limpeza realizada diariamente por sucção.
A temperatura e o oxigênio dissolvido foram mensurados diariamente com oxímetro YSI 55 (Yellow Spring Instrument, Yellow Springs, OH, USA). Os níveis limnológicos de amônia foram mensurados semanalmente, pela reação de Berthelot 
(SOLARANZO, 1969) e nitrito pela reação de Griess (AMINOT; CHAUSSEPIED, 1983).

\section{Vacina}

A vacina foi preparada segundo Pretto-Giordano et al. (2010b). Foi utilizado S. agalactiae (UEL-10) isolado de um surto de estreptococose em tilápias cultivadas em tanque-rede no norte do estado do Paraná.

O cultivo da cepa vacinal foi realizado em meio Tryptic Soy Broth (TSB) (Difco Laboratories, Sparks, MD) e incubado a $30 \pm 1^{\circ} \mathrm{C} / 72 \mathrm{~h}$. Uma alíquota foi utilizada para determinar a concentração bacteriana por espectrofotometria (Modelo Cintra 5), comprimento de onda de $540 \mathrm{~nm}$, densidade óptica (OD) de 0,7860, e contagem em placas (UFC $\mathrm{mL}^{-1}$ ) de Tryptic Soy Agar (TSA) (Difco Laboratories, Sparks, MD). A pureza da cultura foi confirmada por semeadura em Ágar Columbia (Difco Laboratories, Sparks, MD) adicionado de $5 \%$ de sangue ovino (ACS). A cultura em TSB foi inativada por adição de formalina tamponada a $10 \%$, concentração final de $3 \%$, em temperatura ambiente por $48 \mathrm{~h}$. Uma alíquota da cultura tratada foi semeada em ACS para confirmar a inativação das células.

A cultura inativada foi centrifugada a $7000 \mathrm{x}$ g por $30 \mathrm{~min}$, a $10^{\circ} \mathrm{C}$ e o pelet mantido sob refrigeração até o momento do uso. Para a vacinação, $10 \mathrm{~mL}$ do pelet foi diluído em $990 \mathrm{~mL}$ de solução salina estéril a $0,85 \%$ e posteriormente em $9000 \mathrm{~mL}$ de água ultrapura, totalizando $10.000 \mathrm{~mL}$, correspondendo a uma concentração final de 5,4 x $10^{8} \mathrm{UFC} \mathrm{mL}^{-1}$.

\section{Desafio}

O desafio foi realizado com a mesma cepa utilizada na produção da vacina. $S$. agalactiae foi cultivado em $100 \mathrm{~mL}$ de TSB a $30 \pm 1^{\circ} \mathrm{C} / 24 \mathrm{~h}$ e a concentração celular de $3,0 \times 10^{8} \mathrm{UFC}^{-1}$, de acordo com Pretto-Giordano et al. (2010b). O inóculo foi padronizado por espectrofotometria com comprimento de onda de 540nm, OD de 0,7846 e contagem em placas (UFC $\mathrm{mL}^{-1}$ ) com TSA. A pureza do inóculo foi verificada por semeadura em placas de ACS, incubadas a $30 \pm 1^{\circ} \mathrm{C} / 48 \mathrm{~h}$.

\section{Delineamento experimental}

Foram utilizados dois tratamentos e um grupo controle, com três repetições cada (Quadro 1). No tratamento 1 (T1), 140 peixes foram vacinados com uma dose da vacina, administrada por b.i. No tratamento 2 (T2), 134 peixes foram vacinados com duas doses de vacina por b.i. com intervalo de 25 dias. No grupo controle, 147 peixes foram submetidos a b.i. em água ultrapura estéril.

Para a vacinação e banho de água ultrapura, os peixes de cada tratamento foram divididos em grupos de 25 peixes, e cada grupo foi imerso por 20 minutos em $10.000 \mathrm{~mL}$ da solução de vacina ou água ultrapura, com aeração constante e, em seguida, recolocados nas caixas de origem (EVANS et al., 2004b).

Os peixes foram desafiados com $0,1 \mathrm{~mL}$ de inóculo de $S$. agalactiae, com concentração de 3,0 x $10^{8}$ UFC $\mathrm{mL}^{-1}$ por i.p. (PRETTO-GIORDANO et al., 2010b), 43 dias após a aplicação da vacina (Figura 1).

Durante 16 dias após o desafio, os peixes mortos e morimbundosforam retirados diariamente. Dos peixesmortos emoribundos, foramretiradas amostras de encéfalo, rim, fígado, coração, líquido visceral e olhos e semeadas em ACS, incubadas a $30 \pm 1^{\circ} \mathrm{C} / 48$ h. Foi observada a morfologia das colônias e ausência de hemólise e quando consideradas típicas foram submetidas à Coloração de Gram, prova da catalase e esculina. Para a identificação bioquímica foi utilizado o Api 20 Strep (BioMérieux, France) e para a classificação no grupo de Lancefield o Slidex Strepto Kit (BioMerieux, França) (EVANS et al., 2002). 


\section{Estatística}

Para verificar a significância estatística entre os grupos vacinados e controle, utilizou-se o teste de Qui-quadrado corrigido de Yates, com nível de significância de 5\%. O cálculo do Risco Relativo (RR), com intervalo de confiança de 95\%, foi realizado para verificar a força de associação entre a exposição à vacina e o seu efeito protetor. Para os cálculos citados utilizou-se o pacote estatístico Epi6 6,04 (DEAN et al., 1994). A eficácia da vacina foi calculada como porcentagem de sobrevivência relativa (RPS), segundo Amend (1981):

RPS $=1-(\%$ mortalidade dos animais vacinados $/ \%$ mortalidade dos animais controle) $\mathrm{x}$ 100.

\section{Resultados e Discussão}

Durante o experimento, a temperatura foi mantida com média de $23,5^{\circ} \mathrm{C}(19,3-25,1)$ e o oxigênio dissolvido com média de $4,61 \mathrm{mg} / \mathrm{L}$ $(1,17$ - 6,64). A concentração média de amônia foi de $0,22 \mathrm{mg} / \mathrm{L}(0,11-0,86)$ e a de nitrito de $0,029 \mathrm{mg} / \mathrm{L}(0-0,66)$. A mortalidade de peixes no T1 foi de 57 (40,71\%), no T2, $51(38,06 \%)$ e no grupo controle, 76 (51,7\%). Comparativamente ao grupo controle, os peixes do $\mathrm{T} 1$, imunizados com uma dose da vacina, e no T2, imunizados com duas doses, apresentaram os seguintes valores: $\mathrm{p}=0,0805$, $\mathrm{RR}=0,79$ (IC 95\%: 0,61 - 1,01) e RPS $=21,0 \%$ no $\mathrm{T} 1$, e $\mathrm{p}=0,0296, \mathrm{RR}=0,74$ (IC 95\%: 0,56 - 0,96) e RPS $=26,0 \%$. Não houve diferença significativa entre T1 e T2 ( $\mathrm{p}=0,7445)$ (Tabela 2).

Poucos trabalhos foram realizados com vacinas estreptocócicas aplicadas por b.i. e a maioria dos resultados se assemelha aos desta pesquisa. A baixa eficácia da vacina administrada por b.i., quando comparada a outros métodos de inoculação também foi observada por outros pesquisadores (NAKANISHI; KIRYU OTOTAKE, 2002; EVANS et al., 2004 a).

Em 2002, Nakanishi; Kiryu e Ototake (2002) testaram uma vacina de $S$. iniae inativada na concentração de $1,0 \times 10^{9} \mathrm{UFC}^{-1}$ por b.i. em trutas arco íris (Oncorhynchus mykiss) de 4 a $10 \mathrm{~g}$. Os grupos, controle e vacinado, foram desafiados 14 dias após com dose de $2,3 \times 10^{3} \mathrm{UFC}_{\text {peixe }}{ }^{-1}$ por via i.p. A mortalidade foi de $80 \%$ nos dois grupos, demonstrando a ineficácia da via de administração da vacina. Os autores, com a mesma vacina e desafio, imunizaram um grupo de peixes por método que associou micro lesões na pele à b.i. para aumentar a absorção da vacina, e obtiveram taxa de mortalidade de $40 \%$, melhorando a eficácia.

Evans, Klesius e Shoemaker (2004a) pesquisaram uma vacina com 4,0 x $10^{9} \mathrm{UFC} \mathrm{mL}^{-1}$ de células inativada de $S$. agalactiae e concentrado de produtos extracelulares administrada por b.i. No experimento foram vacinados dois grupos de tilápias, um com peixes de $5 \mathrm{~g}$ e outro com $30 \mathrm{~g}$. Os peixes foram desafiados por via i.p. com cepa homóloga na concentração de 3,6 x 105 UFC/peixe, 30 dias após imunização e na concentração de 1,7 x $10^{6} \mathrm{UFC} /$ peixe, 34 dias após, respectivamente. $\mathrm{O}$ RPS foi de $34 \%$ no grupo de $5 \mathrm{~g}$ e $35 \%$ no grupo de $30 \mathrm{~g}$. Os autores concluíram que a via por b.i., apesar do baixo RPS, pode ser considerada satisfatória quando utilizada em larvicultura e em sistemas de produção de juvenis. 
Tabela 2. Eficácia de uma vacina de Streptococcus agalactiae inativado aplicada por banho de imersão em tilápia do Nilo (Oreochromis niloticus) em condições experimentais,Londrina, 2009.

\begin{tabular}{|c|c|c|c|c|}
\hline Tratamentos & Óbitos/Total (\%) & $p^{*}(\leq 0,05)$ & RR (IC 95\%) & RPS\% $^{\mathrm{b}}$ \\
\hline $\mathrm{T1}^{\mathrm{c}}$ & $57 / 140(40,7)$ & 0,0805 & $0,79(0,61-1,01)$ & 21 \\
\hline Controle & $76 / 147(51,7)$ & & & \\
\hline$T 2^{\mathrm{d}}$ & $51 / 134(38,06)$ & 0,0296 & $0,74(0,56-0,96)$ & 26 \\
\hline Controle & $76 / 147(51,7)$ & & & \\
\hline $\mathbf{T} 2^{\mathrm{d}}$ & $51 / 134(38,06)$ & 0,7445 & $0,93(0,70-1,25)$ & \\
\hline$T 1^{c}$ & $57 / 140(40,7)$ & & & \\
\hline
\end{tabular}

* Qui-quadrado corrigido de Yates; ${ }^{\mathrm{a}} \mathrm{RR}$ - Risco relativo; ${ }^{\mathrm{b}}$ Porcentagem relativa de sobrevivência; ${ }^{\mathrm{c}} \mathrm{T} 1$ - peixes vacinados com uma dose; ${ }^{\mathrm{d}} \mathrm{T} 2$ - peixes vacinados com duas doses

Fonte: Elaboração dos autores.

Vacina inativada elaborada com $S$. iniae e Lactococcus garvieae é comercializada na Ásia (AquaVac Garvetil ${ }^{\mathrm{TM}}$, SCHERING-PLOUGH INTERVET, 2007b). Segundo dados do laboratório responsável, a vacinação de alevinos por b.i. com uma dose reforço, incorporada na ração (Aquavac Garvetil $^{\mathrm{TM}}$ Oral), e administrada na fase juvenil apresenta uma eficácia de 50\% contra as bactérias utilizadas na elaboração da vacina. A vacina inativada de $S$. iniae (Norvax ${ }^{\circledR}$ Strep Si, SCHERING-PLOUGH INTERVET, 2007a) administrada por b.i. ou via i.p., segundo o fabricante, quando obedecidas às normas de conservação e uso, pode alcançar eficácia de 68\% e $100 \%$, respectivamente. Shoemaker et al. (2006) avaliaram a eficácia de vacina inativada e liofilizada de $S$. iniae na concentração de $4,0 \times 10^{9}$ células $\mathrm{mL}^{-1}$ administrada por via i.p. e v.o. incorporada à ração utilizando a tecnologia Oralject ${ }^{\mathrm{TM}}$ em tilápias. $\mathrm{O}$ RPS dos peixes vacinados pela v.o. foi de $63,1 \%$ e do grupo vacinado por via i.p. de 100\%. Apesar da vacina protegida ter apresentado menor eficácia o método é menos estressante e possibilita vacinação de grandes populações de peixes.

Outras pesquisas com vacinas inativadas de estreptococos também mostraram que a administração por via i.p. apresentou melhor eficácia. Eldar et al. (1995) em Israel, pesquisaram vacinas constituídas de células inativadas $\left(10^{10} \mathrm{UFC}\right.$ $\left.\mathrm{mL}^{-1}\right)$, de diferentes cepas de $S$. difficile. Os autores utilizaram tilápias de 150 - 180g, vacinadas com duas doses i.p. com intervalo de quatro semanas e desafiadas três semanas após com inóculo de $1,0 \mathrm{x}$ $10^{5}$ UFC peixe $^{-1}$, via i.p. Após 75 dias de observação verificaram que $100 \%$ dos peixes do grupo controle morreram e nos grupos vacinados não ocorreu mortalidade.

Evans et al. (2004b) pesquisaram uma vacina experimental elaborada com S.agalactiae inativado e concentrado de produtos extracelulares por via i.p. em tilápias de 5 e 30 g. Os peixes desafiados por via i.p. com cepa homóloga de Streptococcus agalactiae, 46 e 30 dias após, apresentaram RPS de 25 e $80 \%$, respectivamente.

Pretto-Giordano et al. (2010b) no Brasil, testaram em condições experimentais semelhantes às utilizadas nesse estudo, vacina inativada de $S$. agalactiae por via i.p. com uma e duas doses em tilápias com 20 gramas de peso. Os peixes foram desafiados 30 dias após por via i.p. e o RPS foi de 83,6 e $96,4 \%$, respectivamente.

\section{A vacina Aquavac ${ }^{\circledR}$ STREP Sa (MSD SAÚDE} ANIMAL, 2012) testada no Brasil, apresentou em condições laboratoriais, RPS de $84 \%$ nos peixes vacinados e desafiados com $S$. agalactiae por via i.p. Em condições de campo, a porcentagem de sobrevivência dos peixes vacinados em duas propriedades foi de 91,9 e 87,0 e dos não vacinados de 76,5 e 78,0 , respectivamente. 
Neste trabalho, o início da mortalidade dos peixes, T1, T2 e grupo controle, foram observados a partir do segundo dia após o desafio com pico entre o oitavo e décimo dia. Inicialmente os peixes apresentaram letargia e diminuição de apetite evoluindo para exoftalmia uni e bilateral e posteriormente natação errática, opacidade de córnea, distensão visceral e alteração da coloração de pele. A única diferença observada foi que os peixes dos $\mathrm{T} 1$ e $\mathrm{T} 2$, ao contrário dos do grupo controle, recuperaram gradativamente o apetite a partir do $11^{\circ}$ dia após o desafio.

A cepa utilizada nesta pesquisa, apesar de três passagens prévias em tilápias para reativação, apresentou taxa de mortalidade atípica, entretanto, os sinais clínicos observados foram semelhantes aos descritos na literatura.(EVANS et al., 2002; SALVADOR et al., 2003, 2005; FIGUEIREDO et al., 2006).

Os principais sinais clínicos observados em tilápias com infecção natural ou experimental por Streptococcus spp são típicos de septicemia e meningoencefalite. Os sinais mais freqüentes são: comportamento anormal, natação errática, letargia, exoftalmia uni ou bilateral, opacidade de córnea, anorexia, ascite e escurecimento da pele (PLUMB, 1997, 1999; EVANS et al., 2002). No Brasil, Salvador et al. (2005), Figueiredo et al. (2006), Pretto-Giordano et al. (2010a), também relataram sinais semelhantes em tilápias com infecção natural ou experimental.

Evans et al. (2002) inocularam S. agalactiae isolados de peixes de diferentes regiões naturalmente infectados, em tilápias na concentração de 1,0 x $10^{7}$ UFC peixe ${ }^{-1}$, via i.p. e observaram mortalidade variando de $60 \%$ a $100 \%$, em um período de sete dias após a inoculação.

Evans, Klesius e Shoemaker (2004a) verificaram morbidade e mortalidade de 40 e 50\% em tilápias, nas primeiras 24 horas após inoculação, por via i.p. de $S$. agalactiae nas concentrações de 4,5 x $10^{6}$ e 5,5 x $10^{2} \mathrm{UFC}_{\text {peixe }}{ }^{-1}$, respectivamente.
Pretto-Giordano et al. (2010a) avaliaram experimentalmente a patogenicidade de $S$. agalactiae isolados de infecções naturais em tilápias por inoculação i.p, em concentrações que variaram de $1,0 \times 10^{6}$ a $1,5 \times 10^{8}$ UFC peixe $^{-1}$ e observaram dois picos de mortalidade, um entre o primeiro e segundo dia após a inoculação, e outro entre o sexto e o sétimo dia. As taxas de mortalidade acumulada variaram de 67,5 a $90 \%$, dependendo da concentração do desafio.

Mian et al. (2009) avaliaram a $\mathrm{DL}_{50}$ de cinco cepas de $S$. agalactiae, isoladas de tilápia do Nilo em diversos estados brasileiros e observaram taxas de mortalidade de 90 a 100\%, com apresentação dos primeiros sinais clínicos 24 h após a infecção, e a maior mortalidade com $72 \mathrm{~h}$. Os autores afirmaram que as cepas de S.agalactiae mostraram ser extremamente virulentas para tilápias, necessitando de mais estudos sobre os fatores de virulência e interação agente, hospedeiro e ambiente.

Segundo Chang e Plumb (1996) a intensidade das lesões e sinais clínicos em tilápia depende de fatores relacionados à cepa de $S$. agalactiae, dose infectante, qualidade da água, temperatura, biomassa e manejo.

Os peixes moribundos ou mortos submetidos à necropsia apresentaram como principais lesões macroscópicas: pele esbranquiçada, exoftalmia, opacidade de córnea, presença de líquido amarelado na cavidade visceral, encéfalo hemorrágico, hepatomegalia, esplenomegalia, coração com pontos de fibrose e rim pálido. Estas lesões são compatíveis com as infecções causadas por estreptococos em peixes e são semelhantes às descritas por vários pesquisadores (SALVADOR et al., 2003; HERNANDEZ; FIGUEROA; IREGUI, 2009; INOCENTE FILHO et al., 2009; PRETTOGIORDANO et al., 2010a).

No exame direto e cultivo das amostras de sangue, olho, líquido visceral, fígado, baço, rim e encéfalo com alterações macroscópicas, foi observado a presença de cocos gram positivos 
dispostos em pequenas cadeias. Nas 48/52 amostras biológicas foram isoladas colônias pequenas, não hemolíticas, cocos gram positivos, pertencentes ao grupo B de Lancefield e características fenotípicas correspondentes às descritas para $S$. agalactiae isolados de tilápias (SALVADOR et al., 2005; MIAN et al., 2009). Estas características foram idênticas as da cepa utilizada para a elaboração da vacina e desafio.

\section{Conclusão}

Nas condições deste experimento, os resultados mostraram que a vacina de Streptococcus agalactiae inativado aplicada por banho de imersão em tilápia do Nilo (Oreochromis niloticus) apresentou baixa eficácia.

A vacinação com duas doses da vacina não alterou significativamente a eficácia da vacina.

A cepa de Streptococcus agalactiae utilizada neste trabalho apresentou características de virulência e picos de mortalidade atípicos.

Este trabalho foi registrado e aprovado no Comitê de Ética em Experimentação Animal, sob o número 57/08 (Anexo 1) e realizado de acordo com as normas de biosegurança e ética.

\section{Referências}

AMEND, D. F. Potency testing of fish vaccines. Development in Biological Standardization, New York, v. 49, p. 447-454, 1981.

AMINOT, A.; CHAUSSEPIED, M. Manuel des analyses chimiques em milieu Marin. Brest: CNEXO, 1983.

CHANG, P. H.; PLUMB, J. A. Effects of salinity on Streptococcus infection of Nile tilapia, Oreochromis niloticus. Journal of Applied Aquaculture, Binghamton, v. 6, n. 1, p. 39-45, 1996.

DEAN, A. G.; DEAN, J. A.; COUlOMBIER, D.; BRENDEL, K. A.; SMITH, D. C.; BURTON, A. H.; DICKER, R. C.; SULLIVAN, K. M.; FAGAN, R. F.; ARNER, T. G. Epi info, version 6: a word processing, database, and statistic program for epidemiology on microcomputers. Atlanta: Center for Diseases Control and Prevention, 1994.

DUMRONGPHOL, Y.; HIROTA, T.; KONDO, H.; AOKI, T.; HIRONO, I. Identification of novel genes in japanese flounder (Paralichthus olivaceus) head kidney up-regulated after vaccination with Streptococcus iniae formalin-killed cells. Fish and Shelfish Immunology, Tokyo, v. 26, p. 197-200, 2009.

ELDAR, A.; BEJERANO, Y.; LIVOFF, A.; HOROVITCZ, A.; BERCOVIER, H. Experimental streptococcal meningo-encephalitis in cultured fish. Veterinary Microbiology, Amsterdam, v. 43, n. 1, p. 3340, 1995.

EVANS, J. J.; KLESIUS, P. H.; GILBERT, P. M.; SHOEMAKER, C. A.; AL SARAWI, M. A.; LANDSBER, G. J.; DUREMDEZ, R.; AL MARZOUK, A.; AL ZENKI; S. Characterization of b-hemolytic group B Streptococcus agalactiae in cultured sea bream, Sparus auratus L., and wild mullet, Liza klunzingeri, in Kuwait. Journal of Fish Diseases, Oxford, v. 25, p. 505513, 2002.

EVANS, J. J.; KLESIUS, P. H.; SHOEMAKER, C. A. Efficacy of Streptococcus agalactiae (group B) vaccine in tilapia (Oreochromus niloticus) by intraperitoneal and bath immersion administration. Vaccine, Kidlinton, v. 22, p. 3769-3773, 2004a.

EVANS, J. J.; WIEDENMAYER, A. A.; KLESIUS P. H.; SHOEMAKER, C. A. Survival of Streptococcus agalactiae from fish following natural and experimental infections. Aquaculture, Amsterdam, v. 233, n. 1-4, p. $15-$ $21,2004 \mathrm{~b}$.

FIGUEIREDO, H. C. P.; CARNEIRO, D. O.; FARIA, F. C.; COSTA, G. M. Streptococcus agalactiae associado à meningoencefalite e infecção sistêmica em tilápia do Nilo (O. niloticus). Arquivo Brasileiro Medicina Veterinária e Zootecnia, Belo Horizonte, v. 58, n. 4, p. 678-680, 2006.

FIGUEIREDO, H. C. P.; LEAL, C. A. G. Tecnologias aplicadas em sanidade de peixes. Revista Brasileira de Zootecnia, Viçosa, v. 37, p. 8-14, 2008. Suplemento Especial.

GOMES, S.; AFONSO, A.; GARTNER, F. Fish vaccination againt infections by Streptococcal species and the particular case of Lactococcosis. Revista Portuguesa de Ciências Veterinárias, Lisboa, v. 101, p. 25-35, 2006.

GRISEZ, L.; OLLEVIER, F. Vibrio Listonella anguillarum infections in marine fish larviculture. In: LAVENS, P., JASPERS, E., ROELANDS, I. (Ed.). 
Larvi'91: fish and crustacean larviculture symposium. European Aquaculture Society, Gent, n. 24, special publication, 1995. p. 497.

HASTEIN, T.; GUDDING, R.; EVENSEN, O. Bacterial vaccines for fish-an update of the current situation worldwide. Developments in Biologicals, Basel, v. 121, p. 55-74, 2005.

HERNANDEZ, E.; FIGUEROA, J.; IREGUI, C. Streptococcosis on a red tilapia, Oerochromis sp., farm: acase study. Journal of Fish Diseases, Oxford, v. 32, p. 247-252, 2009.

INOCENTE FILHO, C.; MULLER, E. E.; PRETTOGIORDANO, L. G.; BRACARENSE, A. P. F. R. L. Histological findings of experimental Streptococcus agalactiae infection in Nile tilapias (Oreochromis niloticus). Brazilian Journal of Veterinary Pathology, Botucatu, v. 2, n. 1, p. 12-15, 2009.

KLESIUS, P. H.; EVANS, J. J.; SHOEMAKER, C. A. Warmwater fish vaccinology in catfish production. Animal Health Research Reviews, Wallingford, v. 5, n. 2, p. 305-311, 2004.

MIAN, F. C.; GODOY, D. T; LEAL, C. A. G.; YUHARA, T. Y.; COSTA, G. M.; FIGUEIREDO, H. C. P. Aspects of the natural history and virulence of $S$. agalactiae infection in Nile tilapia. Veterinary Microbiology, Amsterdam, v. 136, n. 1-2, p. 180-183, 2009.

MSD SAÚDE ANIMAL. AQUAVAC® STREP $\mathrm{Sa}$ : resumo da bula. 2012. Disponível em: $<$ http:// www.msd-saude-animal.com.br/products/Copy_of_ AQUAVAC_/020_Resumo_da_Bula.aspx $>$. Acesso em: 2 mar. 2012.

NAKANISHI, T.; KIRYU, I.; OTOTAKE, M. Development of a new vaccine delivery method for fish: percutaneous administration by immersion with application of a multiple puncture instrument. Vaccine, Kidlinton, v. 20, n. 31-32, p. 3764-3769, 2002.

PARK, Y. K.; NHO, S. W.; SHIN, G. W.; PARK, S. B.; JANG, H. B.; CHA, I. S.; KIM, Y. R.; DALVI, R. S.; KANG, B. J.; JUNG, T. S. Antibiotic susceptility and resistence of Streptococcus iniae and Streptococcus parauberis isolated from olive flounder (Paralichthys olivaceus). Veterinary Microbiology, Amsterdam, v. 136, n. 1-2, p. 76-81, 2009.

PLUMB, J. A. Infections diseases of tilapia. In: COSTAPIERCE, B. A., RAKOCY, J. E. Tilapia aquaculture in the Americas. World Aquaculture Society, Baton Rouge, v. 1, p. 212-218, 1997.
Tilapia bacterial diseases. In: Health: maintenance and principal microbial diseases of cultured fishes. Ames: Iowa University Press, 1999. p. 297-305.

PRETTO-GIORDANO, L. G.; MULLER, E. E.; FREITAS, J. C.; SILVA, V. G. Evaluation on the pathogenesis of Streptococcus agalactiae in Nile Tilapia (Oreochromis niloticus). Brazilian Archives of Biology and Technology, Curitiba, v. 53, n. 1, p. 87-92, 2010a.

PRETTO-GIORDANO, L. G.; MULLER, E. E.; KLESIUS, P.; SILVA, V. G. Efficacy of an experimental inactivated Streptococcus agalactiae vaccine in Nile tilapia (Oreochromis niloticus) reared in Brazil. Aquaculture Research, Oxford, v. 41, n. 10, p. 15391544, 2010b.

ROBERTS, R. J.; SOMMERVILLE, C. Diseases of tilapias. In: PULLIN, S. V.; LOWE-MAC CONNEL, R. H. (Ed.). The biology and culture of tilapias. Manila: International Center for Living Aquatic Resources Management, 1982. p. 247-263.

SALVADOR, R.; MULLER, E. E.; FREITAS, J. C.; LEONHADT, J. H.; PRETTO-GIORDANO, L. G.; DIAS, J. A. Isolation and characterization of Streptococcus spp. group B in Nile tilapia Oreochromis niloticus) reared in hapas nets and earth nurseries in the northern region of Parana State, Brazil. Ciência Rural, Santa Maria, v. 35, p. 1374-1378, 2005.

SALVADOR, R; MÜLLER, E. E.; LEONHARDT, J.H.; PRETTO-GIORDANO, L. G.; DIAS, J. A.; FREITAS, J. C.; MORENO, A. M. Isolamento de Streptococcus spp. de tilápia do Nilo (Oreochromis Niloticus) e qualidade da água de tanques rede na Região Norte do Estado do Paraná, Brasil. Semina: Ciências Agrárias, Londrina, v. 24, n. 1, p. 35-42, 2003.

SCHERING-PLOUGH INTERVET ANIMAL HEALTH - AQUACULTURE. Disease prevention: Norvax ${ }^{\circledR}$ Strep Si Asia. 2007a. Disponível em: <http:// spaquaculture.com/assets/Garvetil.pdf.>. Acesso em: 15 fev. 2010.

Total protection strategies against streptococcosis in farmed tilapia: AquaVac ${ }^{\mathrm{TM}}$ Garvetili ${ }^{\mathrm{TM}}$, AquaVac ${ }^{\mathrm{TM}}$ Garvetil ${ }^{\mathrm{TM}}$ Oral. 2007b. Disponível em: $<$ http://spaquaculture.com/assets/Garvetil.pdf.>. Acesso em: 29 mar. 2007.

SERRANO, P. H. Responsible use of antibiotics in aquaculture. Food and Agriculture Organization (FAO) Fisheries Technical Paper, Roma, n. 469, p. 11-38, 2005.

SHOEMAKER, C. A.; EVANS, J. J.; KLESIUS, P. H. Density and dose: factors affecting mortality of Streptoccus iniae infected tilapia (Oreochromis niloticus). Aquaculture, Amsterdam, v. 188, p. 229-235, 2000. 
SHOEMAKER, C. A.; VANDERBERG, G. W.; DESOMEAUX, A.; KLESIUS, P. H.; EVANS, J. J. Efficacy of a Streptococcus iniae modified bacterin delivered using Oralject ${ }^{\mathrm{TM}}$ technology in Nile tilapia (Oreochromis niloticus). Aquaculture, Amsterdam, v. 255, p. 151-156, 2006.

SOLARANZO, L. Determiantion of ammonia in natural waters by the phenolhypoclorite method. Limnology and Oceanographfy, Canadá, v. 14, n. 5, p. 799-801, 1969.
SWAIN, P.; NAYAK, S. K.; SAHU, A.; MOHAPATRA, B. C.; MEHER, P. K. Bath immunization of spawns, fries and fingerlings of Indian major carps using a particulate antigen and determination of age, dose and duration of antigen exposure. Fish and Shellfish Immunology, London, v. 13, p. 133-140, 2002.

TORANZO, A. E.; MAGARIÑOS, B.; ROMALDE, J. L. A review of the main bacterial fish diseases in mariculture systems. Aquaculture, Amsterdam, v. 246, n. 1-4, p. 3761, 2005. 